\title{
A reappraisal of the relationship between arachnoid cysts of the middle fossa and chronic subdural haematoma
}

\author{
A PAGE, R M PAXTON, D MOHAN* \\ From the Departments of Radiology and Neurosurgery, ${ }^{*}$ Freedom Fields Hospital, Plymouth, UK
}

SUMMARY It has been increasingly recognised that patients with arachnoid cysts of the middle fossa appear more susceptible to the development of subdural haematomas. Seven patients with arachnoid cysts of the middle fossa and associated subdural haematomas are presented. Intra-cystic haemorrhage, masking the presence of an arachnoid cyst on computed tomography (CT) is highlighted. Repeat of CT scanning in young patients with subdural haematomas in the absence of severe trauma is recommended. Two theories are proposed to account for the observed susceptibility to the development of subdural haematomas in these patients.

Arachnoid cysts are fluid filled cavities within the arachnoid or between the arachnoid and pia mater. They most commonly occur in the middle fossa, and in such a position are often considered as a separate clinical entity. Characteristic plain radiographic, ${ }^{1-4}$ computed tomographic, ${ }^{5-9}$ and invasive investigative appearances $^{1710}$ are recognised.

The mechanism of formation was reviewed by Robinson in $1971^{1}$ and can be either primary developmental or secondary. Further study has not changed this view to date.

The association between arachnoid cysts and subdural haematomas has been noted. ${ }^{1.71011}$ It has been suggested that the presence of an arachnoid cyst in the middle fossa makes the patient more susceptible to developing a subdural haematoma. ${ }^{101213}$ There is often a history of mild trauma but subdural haematomas may arise idiopathically.

Seven cases of arachnoid cysts of the middle fossa with associated subdural haematomas are presented. The possible significance of these cysts is discussed with special reference to the association with subdural haematomas.

Address for reprint requests: Dr AC Page, Department of Radiology, Freedom Fields Hospital, Plymouth, Devon PL4 8NL, UK.

Received 3 June 1986. Accepted 15 August 1986

\section{Case reports}

\section{Case 1}

A 15 year old male was admitted with a 4 week history of right parietal headache, 3 days vomiting and increasing paraesthesia involving the left arm. Three months previously he had been kicked over the left parietal region and as a result struck his right parietal region against a wall. Clinical examination revealed bilateral papilloedema, nystagmus and a slow pulse. Plain skull radiographs showed expansion of the right middle fossa. Computed tomography (CT) demonstrated a right middle fossa arachnoid cyst with a large subdural haematoma and midline shift to the left. At craniotomy a large chronic subdural haematoma extending from the frontal to the occipital pole and overlaying the arachnoid cyst on the right was found. There was a haematoma within the cyst. All dural membrane surrounding the haematoma and part of that of the arachnoid cyst was removed. There was steady clinical improvement paralleled by radiological resolution and a reduction in the size of the arachnoid cyst. The latter was no longer visible on CT scan 3 years later.

\section{Case 2}

A 17 year old male was referred with a history of a few days of increasing occipital headache radiating to the back of the neck, and intermittent episodes of vomiting. He had been known to have suffered severe headaches at approximately monthly intervals for some years. No record of head injury was given on direct questioning. Clinical examination was unremarkable, plain film radiography of the skull revealed enlargement of the right middle fossa. CT scan showed an arachnoid cyst in the right middle fossa with a subdural haematoma lying superficial to it. Haemorrhage was noted in the arachnoid cyst. Operation confirmed the above findings showing a chronic haematoma overlying an arachnoid cyst. 
A small communication between the cyst and subarachnoid space inferiorly was noted. The cyst fluid was thick and heavily blood stained. The haematoma was evacuated and the membranes excised. Clinical recovery was satisfactory and no residual neurological deficit resulted.

\section{Case 3}

An 11 year old boy was admitted with frontal headache, diplopia and vomiting. He had sustained a mild head injury 2 weeks previously. Bilateral papilloedema was found on clinical examination, plain radiographs of the skull showed no abnormality but a CT scan revealed a left chronic subdural fluid collection (fig 1). Blood stained fluid was drained through burr holes. The subdural fluid re-accumulated and there was recurrence of the symptoms, hence a subdural peritoneal shunt was inserted, unfortunately needing revision on two subsequent occasions. Following further reaccumulation of fluid with an associated increase in the midline shift of CT scan, a left temporal craniectomy with excision of the subdural membrane and removal of the shunt was performed. Subsequently an area of decreased density was seen in the left temporal region on a CT scan 7 months later (fig 2). This had not been visible on five previous scans and had the characteristics of an arachnoid cyst. Further craniectomy was performed and the cyst was excised; histological sections of the cyst wall confirmed the diagnosis. At the present time the patient has occasional post orbital pain

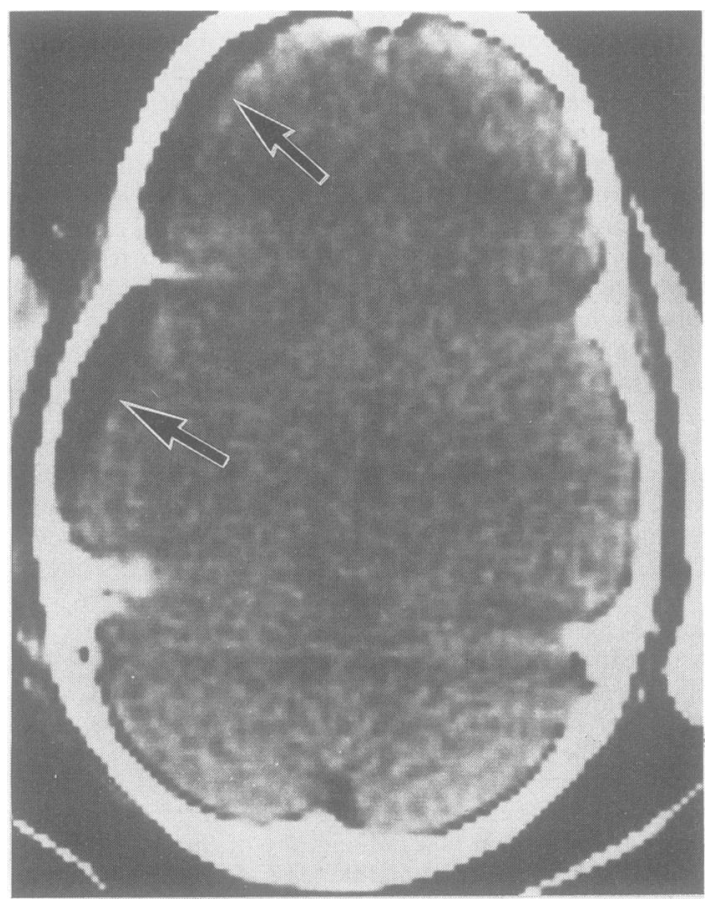

Fig 1 Admission CT scan $(L+44, W 75)$ of a case no 3 showing a chronic subdural haematoma over most of the cerebral cotex (arrowed), with midline shift to the right.

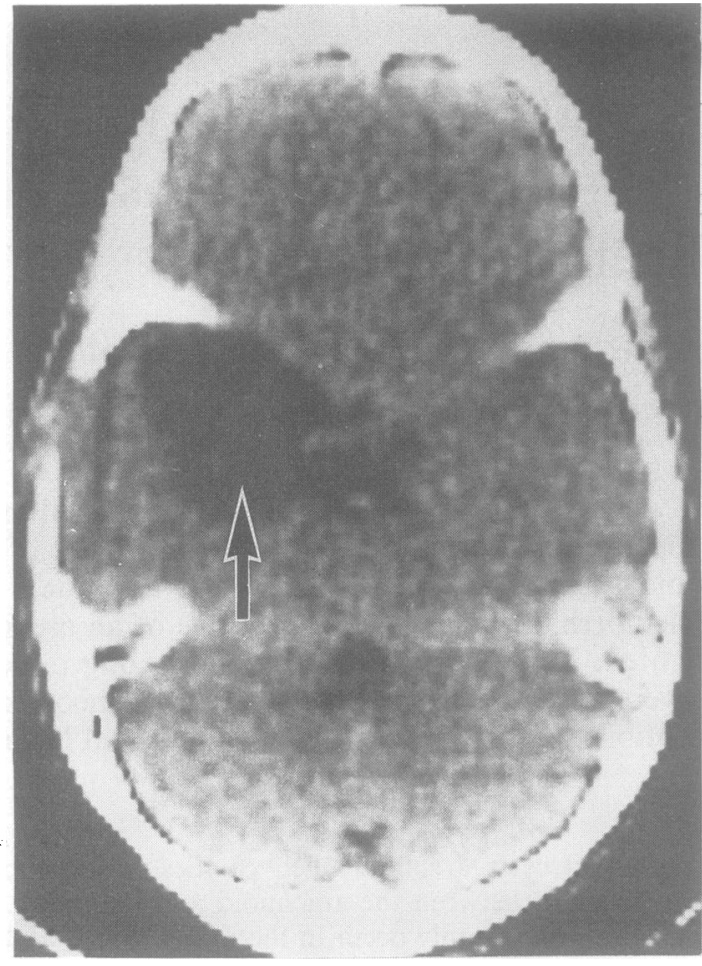

Fig 2 Seventh follow up CT scan $(L+44, W 75)$ on case no 3 revealing for the first time a large low density area in the left anterior temporal region (arrowed). This is seen to extend to the chiasmatic cistern.

and shows no signs of raised intracranial pressure. Cranioplasty was successfully conducted with no complications.

\section{Case 4}

A 23 year old woman was admitted with morning headaches and nausea following a head injury 3 weeks previously. There was early papilloedema but no neurological signs on clinical examination. Plain skull films (fig $3 a$ and $b$ ) and a CT scan (fig 4), showed an enlarged right middle fossa, there being an arachnoid cyst with accompanying bilateral chronic subdural haematoma. There was midline shift to the left. Burr holes were made and a high pressure chronic subdural haematoma was drained and a cysto-peritoneal shunt carried out. The fluid drained was cloudy and blood stained on the right, cloudy and yellow on the left. Clinical resolution was good, CT scan showing reduction of cyst size and no midline shift 3 months after presentation. Subsequent CT scans from 18 months after presentation to date show a residual small cyst with no alteration in characteristics over this time.

\section{Case 5}

A 57 year old barrister presented having been thrown from a horse 7 days prior to admission suffering 30 seconds traumatic amnesia. He complained of bifrontal headache and 

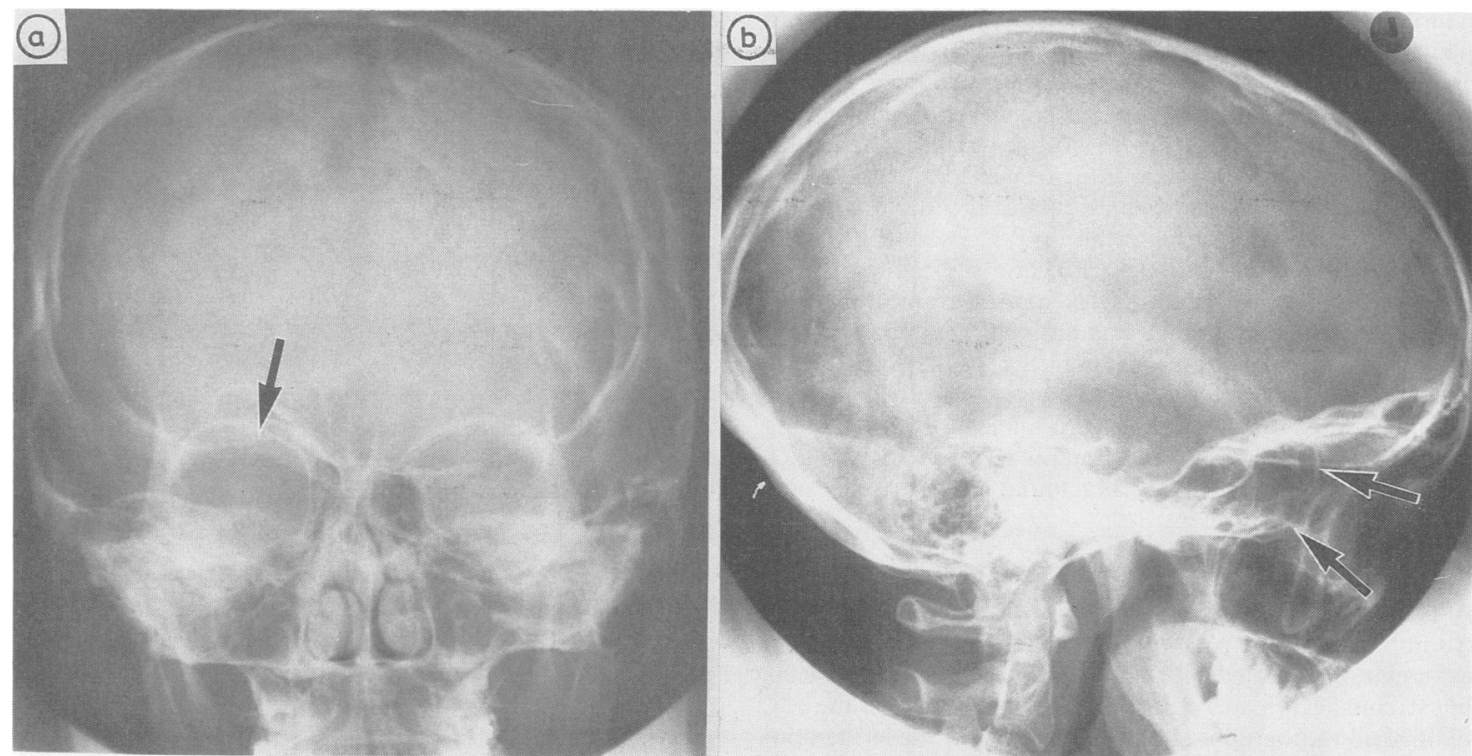

Fig 3 (a and b) PA and lateral skull radiographs of case no 4. Arrows point to the enlarged right middle fossa.
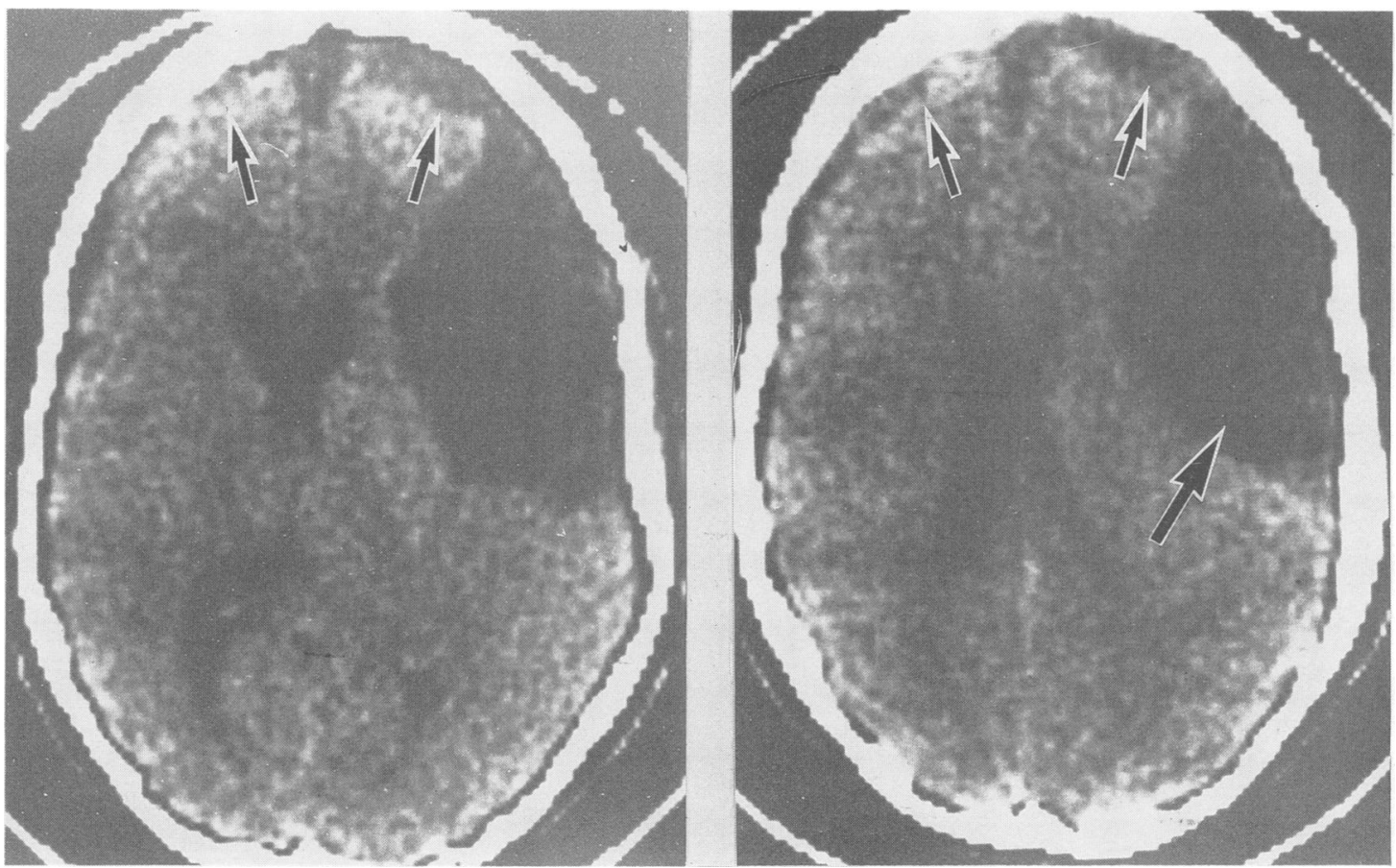

Fig $4 C T$ scan $(L+44, W 75)$ of case no 4, demonstrating an arachnoid cyst in the enlarged middle fossa (large arrows), and bilateral chronic subdural haematomas (small arrows). 
nausea. Examination showed no neurological deficit and plain skull radiographs were normal. CT scan (fig 5) showed an arachnoid cyst in the left middle fossa and a shallow chronic subdural haematoma over the right hemisphere. Slight supra-tentorial shift from left to right was noted. Subsequently, the patient's symptoms worsened and follow up CT scan showed a large isodense subdural collection on the left, the arachnoid cyst being no longer visible (fig 6). The shift to the right was more marked and the right subdural collection no longer visible. A left temporal craniectomy was conducted and yellow fluid was drained from the cyst and subdural space. The cyst was operatively connected to the chiasmatic system and the subarachnoid space over the left cerebral hemisphere. At present this man is still complaining of headache, relieved by analgesia although CT scans (fig 7), show mild improvement, the arachnoid cyst became visible again following treatment.

\section{Case 6}

A 17 year old male was admitted with a 7 day history of frontal headache, photophobia and vomiting. Clinical examination revealed bilateral papilloedema, neck stiffness in extreme flexion and a positive Babinski reflex on the left. Plain skull radiographs showed expansion of the left temporal fossa with elevation of the sphenoid ridge. CT scan revealed a chronic subdural haematoma over the left hemisphere extending to the temporal fossa, with associated midline shift to the right (fig 8). At operation the haematoma was drained by burr holes. There was satisfactory post operative progress, the patient having no residual neurological deficit. Subsequently follow up CT scan showed a low den-
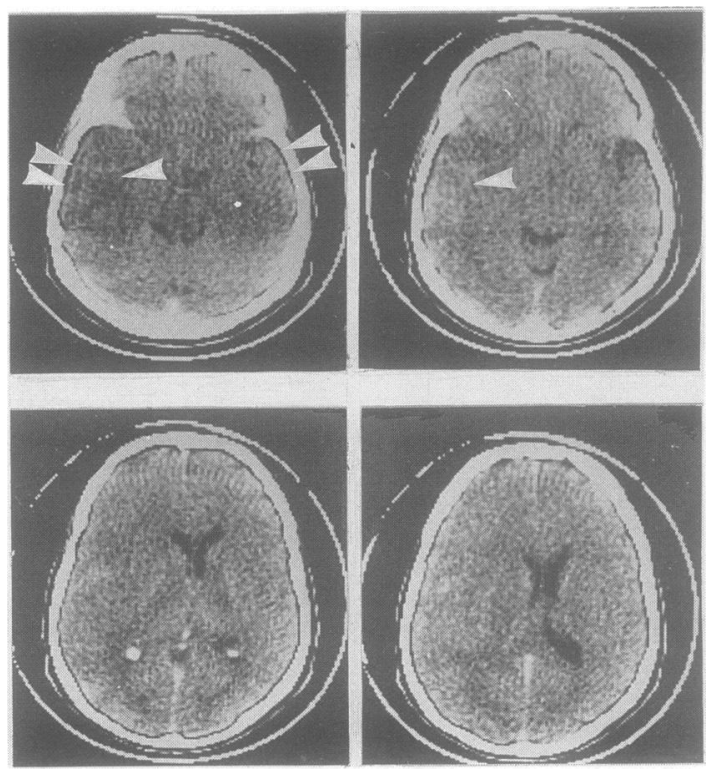

Fig $6 C T(L+44, W 75)$ of case no 5 approximately six weeks after first presentation. The arachnoid cyst of the middle fossa of the left side is now masked by intracystic isodense haemorrhage (single arrow). There is increased midline shift to the right indicating an isodense subdural haematoma (double arrows).
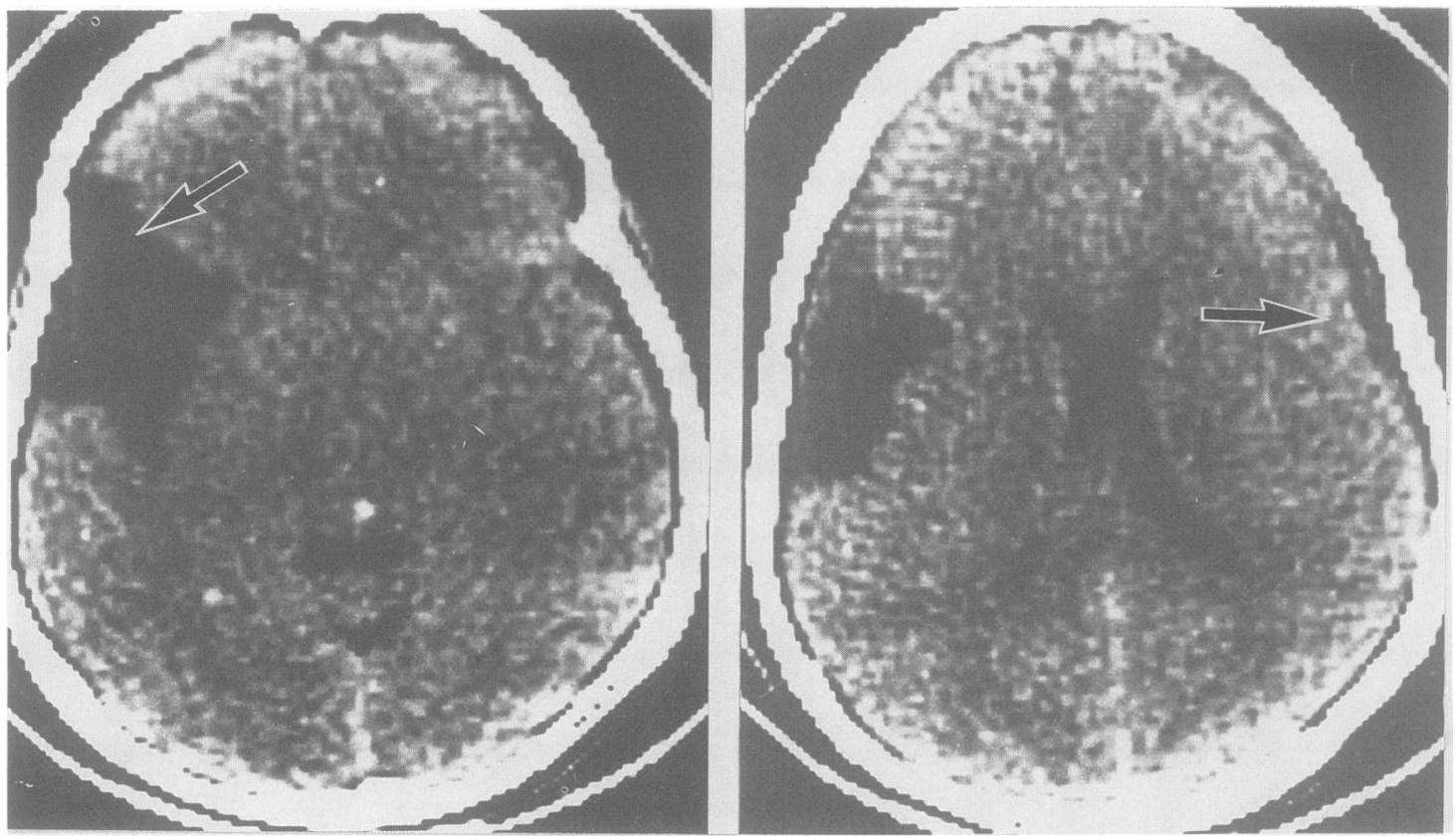

Fig 5 Admission CT scan ( $L+44, W 75)$ on case no 5. An arachnoid cyst of the middle fossa on the left is seen (small arrow'), and a shallow chronic subdural haematoma is seen over the right hemisphere (large arrows). 

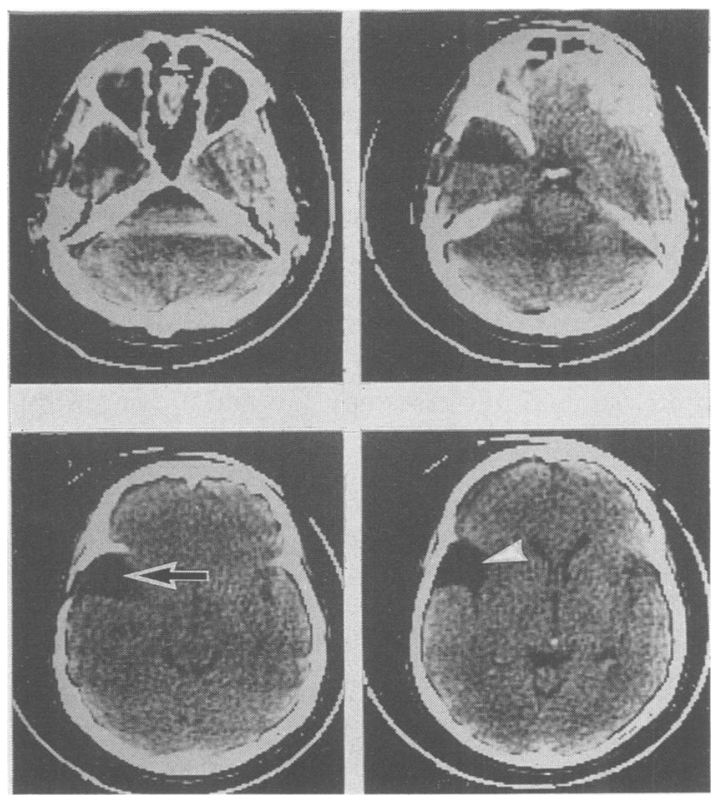

Fig 7 CT scan $(L+44, W 75)$ of case no 5, five weeks post craniectomy. The arachnoid cyst (arrowed) is again visible in the left middle fossa. The midline shift seen in fig 6 is resolving.

sity area in the left temporal fossa consistent with an arachnoid cyst (fig 9). This appeared to shrink in size on CT scan accompanying a steady resolution of the midline shift. Two and a half years later the arachnoid cyst was still present, there being minimal residual midline shift. The patient is at present fit and well.

\section{Case 7}

A 12 year old girl presented with a 2 month history of headache and associated vomiting. There was no history of trauma. The past medical history was unremarkable. Clinical examination revealed papilloedema, there were no abnormal neurological signs. Plain skull radiographs showed expansion of the middle fossa and no evidence of fracture. CT scanning demonstrated an isodense subdural haematoma with a cystic lesion of CSF density being seen in the enlarged left middle fossa, consistent with an arachnoid cyst. The subdural was drained operatively, blood stained fluid being found. The post operative progress was satisfactory.

\section{Discussion}

Arachnoid cysts of the middle fossa were reviewed by Robinson. ${ }^{1}$ They commonly present below the age of 20 years although they can be found at any age. They occur in a ratio of at least 4-1 male to female, being rarely found in females over the age of 20 years. Arachnoid cysts of the middle fossa are found more commonly on the left than the right.

The patients usually present either with a skull deformity as a result of the cyst within the calvarium (as is commonly the case in children), or with the symptoms of raised intracranial pressure following a complication of an arachnoid cyst of the middle fossa. Plain skull radiography may or may not show evidence of enlargement of the middle cranial fossa. Computed tomography (CT) demonstrates a region in the anterior portion of the middle cranial fossa which has attenuation values equivalent to that of CSF and additional features as outlined by Banna. ${ }^{5}$

Arachnoid cysts of the middle fossa are generally considered to be a congenital anomaly. ${ }^{141014}$ Starkman etal ${ }^{14}$ demonstrated four necropsy cases of arachnoid cyst which had shown no symptoms related to the arachnoid cysts of the middle fossa antemortem. They showed the cyst to be surrounded completely by arachnoid in three, and to have an outer membrane of arachnoid and an inner of pia mater in the remaining one. This has been confirmed by other authors, ${ }^{15}$ and shown in our cases 3 and 5, although at operation it is difficult to obtain sections of the inner lining membrane. Starkman ${ }^{14}$ proposed a diverticulum or false passage being created at the time of CSF space formation in foetal development. It is believed that pulsatile CSF flow originating from the choroid plexus is responsible for the CSF space development by invaginating the perimedullary mesenchyme. Thus if a local alteration of CSF flow dynamics occurred during CSF space development, ${ }^{1617}$ it
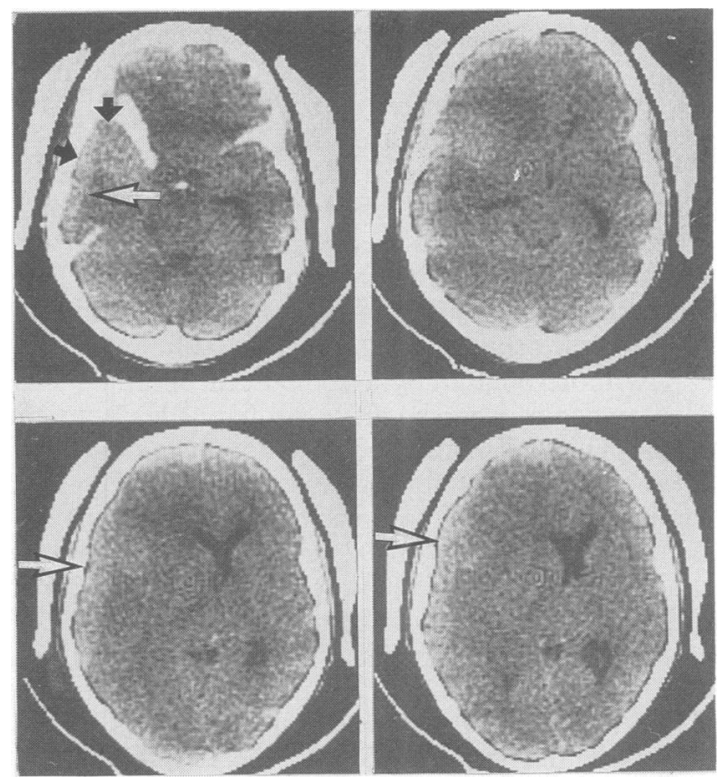

Fig 8 Admission CT scan $(L+44, W 75)$ of case no 6.

The left temporal fossa is enlarged (small arrows). A chronic subdural haematoma is present over the left hemisphere (large arrows). 


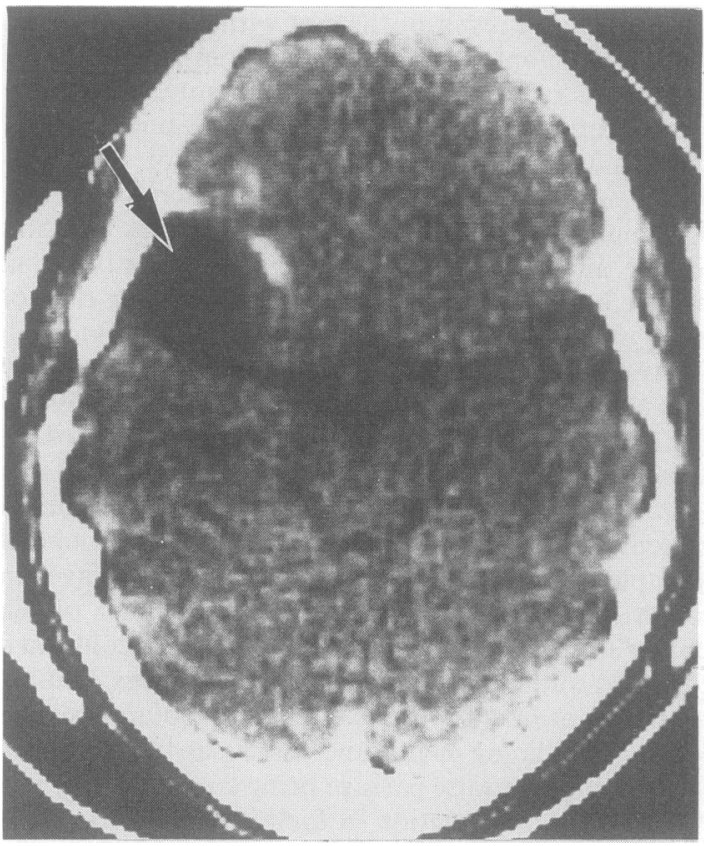

Fig 9 CT scan after subdural haematoma drainage $(L+$ $44, W 75$ ) of case no 6. A low density area of CSF density is now seen in the left middle fossa (arrows).

would seen reasonable to suggest that CSF could become sequestered within the forming arachnoid or pia or both. Clinical support for this theory has been gained by the demonstration of cysto-subarachnoid communications, usually found in the basal cisterns $^{1318}$ and in our case 2 .

It has been suggested that minor intra-uterine trauma to the temporal lobe tip may lead to the development of arachnoid cysts, ${ }^{4}$ in combination with damage to the anterior part of the temporal lobe. Robinson ${ }^{13}$ suggested that agenesis of the temporal lobe was the primary developmental fault; however, it is now more commonly accepted that multiple local developmental abnormalities probably leads to the development of an arachnoid cyst in the middle fossa.

Secondary arachnoid cysts can also be created. These may follow trauma, usually major, occasionally minor or following infection. ${ }^{1}$ Mechanisms cited included the formation of oedema leading to splitting of the arachnoid resulting in the leak of CSF or the trapping of pockets of arachnoid containing CSF following trauma. The formation of adhesions following infection may lead to loculation of CSF.

A potential susceptibility to the development of subdural haematoma in patients with arachnoid cysts of the middle fossa was recognised initially by Robinson ${ }^{1}$ and reiterated by Smith and Smith. ${ }^{10}$ The complications occurring in patients with arachnoid cysts of the middle fossa are illustrated by the cases presented in this paper, and by other authors. ${ }^{179-111519}$ These may be recognised on a CT scan by either midline shift, a subdural haematoma, or intracystic haemorrhage. Cyst rupture and associated subdural haematoma formation have been shown by our cases 1 and 4 and by Kushino ${ }^{12}$ and Lesoin et al. ${ }^{19}$ Intracystic haemorrhage has been demonstrated $^{12}$ and is shown in three cases on CT in this study. La Cour et ll $^{7}$ hypothesised the potential masking of an arachnoid cyst by intracystic haemorrhage and our cases nos 3,5 and 6 demonstrate this.

The occurrence of intracystic haemorrhage may be more common than at first realised, five out of seven patients in our study having demonstrable haematoma within the cyst at operation. In a patient who is found to have midline shift and a suspected isodense subdural haematoma, the presence of enlargement of the middle fossa of the affected side or the contralateral side should always raise the suspicion of an arachnoid cyst being an underlying potential predisposing factor.

The benefit of recurrent follow up CT scans is clearly demonstrated in our cases 3 and 6 where the arachnoid cysts only became visible some time after the initial treatment had been instituted. It is recommended that in young patients with subdural haematoma with no obvious predisposing factors, repeat CT should be conducted.

No satisfactory explanation has been given to explain the susceptibility of patients with an arachnoid cyst of the middle fossa to develop subdural or intracystic haematomas. We propose two theories to explain this:

(1) In response to mild trauma, flow changes within the CSF could be magnified by the arachnoid cyst leading to rupture of bridging veins or vessels in the cyst wall. It is recognised that pressure is transferred more readily and is magnified through the cyst fluid in comparison with the normal subarachnoid CSF space. This has been used to explain the calvarial enlargement and thinning overlying some arachnoid cysts of the middle fossa. ${ }^{1}$ In the development of growing fractures in children ${ }^{20} \mathrm{CSF}$ pressure wave magnification through leptomeningeal cysts is an accepted mechanism of their formation.

(2) The arachnoid cyst is less compliant than normal brain resulting in reduced intra-calvarial cushioning following minor trauma. Thus haemorrhage may occur from bridging veins ipsilaterally or contralaterally resulting in subdural haematomas. Ipsilateral subdural haematoma occurrence is well recognised, contra-lateral haematomas were found in cases 4 and 5 in our study, case 2 of Weinberg and Flom $^{11}$ and in two cases cited by Wortmann. ${ }^{21}$ 
Further clinical and experimental study is needed to confirm these hypotheses.

We are grateful to $\mathrm{Mr} \mathrm{H}$ Gossman and $\mathrm{Mr}$ WE Strachan for allowing us to include their patients in this study. We thank Miss K Roberts for typing the manuscript.

\section{References}

1 Robinson RG. Congenital cysts of the brain: arachnoid malformations. Progr Neurosurg 1971;4:133-74.

2 Davidoff LM, Dyke CG. Relapsing juvenile chronic subdural haematomas. Bull Neurol Institute. New York. 1938;7:95-111.

3 Bull JWD. The diagnosis of chronic subdural haematoma in children and adolescents. $B r J$ Radiology 1949;22:68-80.

4 Tiberin P, Gruskiewitz J. Chronic arachnoidal cysts of the middle fossa and their relation to trauma. $J$ Neurol Neurosurg Psychiatry 1969;24:86-91.

5 Banna M. Arachnoid cysts on CT. Am J Roentgenol 1976;127:979-82.

6 Dyke P, Gruskin P. Supratentorial arachnoid cysts in adults. Arch Neurol 1977;34:276-79.

7 La Cour F, Trevor R, Carey M. Arachnoid cysts and associated subdural haematomas. Arch Neurol 1978; 35:84-89.

8 Anderson FM, Segall HD, Caton WL. Use of computerised tomography scanning in supra temporal arachnoid cysts. J Neurosurg 1979;56:333-8.
9 Leo JS, Pinto RS, Hulvat GF, Epstein F, Kircheff II. Computed tomography of arachnoid cysts. Radiology 1979;130:675-80.

10 Smith RA, Smith WA. Arachnoid cysts of the middle fossa. Surg Neurol 1976;5:246-52.

11 Weinberg PE, Flom RA. Intracranial subarachnoid cysts. Radiology 1973;106:329-33.

12 Kusuno K, Yoshida Y, Takahashi A, Ishii S. Chronic subdural hygroma caused by rupture of arachnoid cyst. Neurol Med Chir Tokyo 1984;24:349-54.

13 Robinson RG. The temporal lobe agenesis syndrome. Brain 1964;87:87-106.

14 Starkman SP, Brown TC, Linell EA. Cerebral arachnoid cysts. J Neuropathol Exp Neurol 1958;17:484-500.

15 Akardi J, Baumann F. Supratentorial extracerebral cysts in infants and children. J Neurol Neurosurg Psychiatry 1975;38:57-68.

16 Flexner L. Contribution of embryology No 110. Publication No 394. Washington: Carnegie Institute, 1929;20:31.

17 Weed L. Certain anatomical and physiological aspects of the meninges and CSF. Brain 1935;58:383.

18 Nagoulitch I, Petrovitch M. Les collections liquidienne extra-cerebrales chez l'enfant. Lyon Chir 1960;56: 726-39.

19 Lesoin F, Dhellemmes P, Russeaux M, Jomin M. Arachnoid cysts and head injury. Acta Neurochirurgica 1983;69:43-51.

20 Editorial. Growing fractures of the skull. Br Med $\mathrm{J}$ 1978;2:519.

21 Wortmann V. Zur Frage des Chronischen Subdura Hydroms. Zentralbl Chir (Liepzig) 1954;79:1620-6. 